\title{
A Modified PTS Scheme for PAPR Reduction in OFDM System with Low Complexity
}

\author{
Monica Kathuria \\ Deptt. Of ECE \\ MIT \\ Moradabad, India
}

\author{
Pankaj Sharma \\ Deptt. Of ECE \\ MIT \\ Moradabad, India
}

\author{
Umesh Gupta \\ Deptt of ECE \\ Meri College of Engg. \&Tech \\ Sampla, India
}

\begin{abstract}
Orthogonal Frequency Division Multiplexing (OFDM) is the most desired and attractive among the various broadband wireless communication system because of its robustness against multipath fading. However even after its so many advantages OFDM has been able to deliver upto its potential due to its "High Peak to Average Power Ratio (PAPR)" which causes power inefficiency in RF section of the transmitter effecting the throughput. The soul purpose of this paper is to provide the most effective PAPR reduction technique while doing amendments in the Partial Transmit Sequence and is termed as the modified Partial Transmit Sequence which overcomes the limitation of conventional system in which a property of cyclically shifting of IFFT is used which doesn't require the side information and provides a system with low complexity.
\end{abstract}

\section{Keywords}

PAPR, PTS, IFFT, CCRR.

\section{INTRODUCTION}

Orthogonal frequency division Multiplexing (OFDM) is a Multicarrier modulation technique where multiple data streams are modulated with mutually orthogonal carrier. In OFDM a high rate data stream is divided into a various number of lower rate data streams which would eventually get transmitted over a number of subcarriers simultaneously [1]. These subcarriers are overlapped with each other in order to increase the spectral efficiency hence providing desired resource utilization. One of the major attractive and effective feature of OFDM is its efficiency in utilization of the available network spectrum also stating its capability to effectively utilize the various other desired resources. Among the different effective and advantageous attributes some of the most popular are as its immunity to the intersymbol interference, its robustness with respect to multipath fading and ability for effectively transfer high data rates. Due to its high spectrum efficiency and channel robustness it is used in digital audio/videos for high speed communication .The major drawback of OFDM is high PAPR which causes nonlinear distortion. Many techniques has been designed to overcome this PAPR issue in which conventional PTS is an attractive and most adopted method [2].

In this paper we proposed modified partial transmit sequence to reduce the peak-to-peak-average ratio (PAPR) in orthogonal division multiplexing (OFDM) system. The proposed technique uses the cyclically shifting property of IFFT which doesn't require the explicit transmission of side information which is required in conventional partial transmit sequences (PTS) technique [3]. The paper is comprised of following sections: In section II an introduction of PAPR in OFDM system has been highlighted which is followed by the proposed method in section III in which conventional and modified Partial Transmit Sequence is shown for improving the computational complexity an analysis has been done in this section. Its results are shown in section IV which is carried on MATLAB.This is followed by the conclusion in section $\mathrm{V}$.

\section{PAPR IN OFDM SYSTEM}

Consider the $\mathrm{N}$ input blocks of length $\mathrm{N}$ which is given as $\left(x_{n}, n=0,1, \ldots \ldots N-1\right)$.Each symbol modulates a single set of subcarriers $\left(f_{n}, n=0,1,2, \ldots N-1\right)$. The chosen subcarriers are orthogonal and given as $f_{n}=n \Delta f$ in frequency domain where $\Delta f=1 / N T, \mathrm{~T}$ is the duration of symbol. Thus, the transmitted OFDM signals is given as:

$$
\chi(\mathrm{t})=\frac{1}{\sqrt{N}} \sum_{k=0}^{N-1} X_{k} e^{j 2 \pi f_{k} t}, 0 \leq t \leq N T
$$

Where $j=\sqrt{ }(-1)$, Figure 1 shows the block diagram of OFDM system.

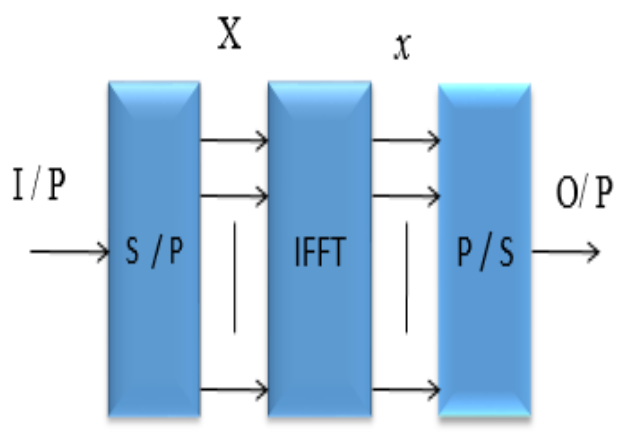

Figure1. Block diagram of basic OFDM system

PAPR in OFDM signal is defined as the ratio of maximum instantaneous power and its average power which is given as:

$$
P A P R=\frac{\max |x(t)|^{2}}{E|x(t)|^{2}}
$$

Where $\mathrm{E}$ denotes the expectation operator.PAPR and in $\mathrm{dB}$ it is calculated and given as $P A P R=10 \log _{10}(P A P R)$. 


\section{PROPOSED METHOD}

\subsection{Conventional Partial Transmit Sequence (C-PTS)}

Conventional Partial Transmit Sequence C-PTS is the most halsen technique for the reduction of PAPR in OFDM system. The PTS model is shown in figure in 2 .X denotes the data block and given as a vector:

$$
X=\left[X_{0}, X_{1}, \ldots \ldots X_{N-1}\right]^{T}
$$

The above vector $\mathrm{X}$ is divided into $\mathrm{M}$ disjoint subblocks and represented by vector:

$$
\begin{aligned}
& \left\{X_{m}, m=1,2,3 \ldots . M\right\} \\
& X=\sum_{m=1}^{M} X_{m}
\end{aligned}
$$

All the subcarrier that are presented in another subblock. An IFFT is employed as:

$$
x=\operatorname{IFFT}\{X\}
$$

Applying phase rotation sector to each subblock:

$$
b_{m} \in\{ \pm 1, \pm \mathrm{j}\}
$$

Where $b_{m}, m=1,2,3 \ldots . M$, we can set weighting factor $b_{1}=1$, where the optimum phase sequence is analyzed which minimizes PAPR of the OFDM signal[4,5], then-

$$
\begin{aligned}
x^{\prime} & =\left[x_{1}, x_{2}, \ldots x_{m}\right]\left[\begin{array}{c}
b_{1} \\
b_{2} \\
\vdots \\
b_{m}
\end{array}\right] \\
x^{\prime} & =\sum_{m-1}^{M} b_{m} x_{m}
\end{aligned}
$$

And $\left\{b_{1}, b_{2}, \ldots \ldots \ldots . . ., b_{m}\right\}=\arg \min \left[\max \left|\sum_{m-1}^{m} b_{m}, x_{m}\right|^{2}\right]$

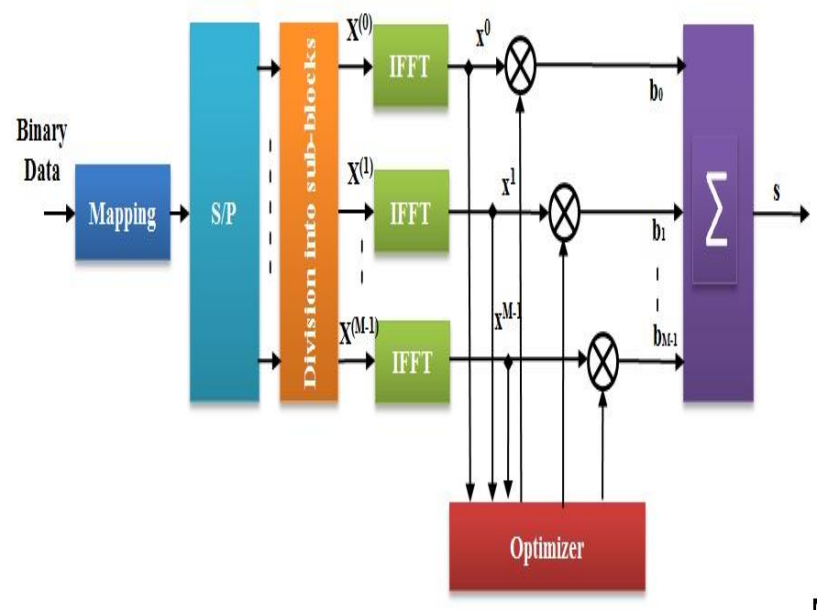

Figure 2. Block diagram of Conventional PTS

\subsection{Modified PTS Scheme (M-PTS)}

This scheme is the amended version of conventional PTS scheme as it lowers the complexity of the transmitter and the receiver without the help of side information Unlike the C-PTS ,this modified version generates a sequence by cyclically shifting the signals and then combining them together and given as [6,7]:

$$
\bar{x}=\sum_{m=1}^{M} \bar{x}^{m}(k)
$$

Where the shifting number is $\boldsymbol{k}$, for sub-block signals $x^{m}$

$$
\begin{aligned}
& \text { and } \bar{x}^{m}(k) \text { is: } \\
& \begin{aligned}
\bar{x}^{m}(k) & =\operatorname{circular}\left(x^{m}, k\right) \\
& =\left[x^{m}(k), \ldots, x^{m}(L N-1), x^{m}(0), \ldots, x^{m}(k-1)\right]
\end{aligned}
\end{aligned}
$$

For directly computing the signal the equation we can use the equation (8) when they have only different in sub-block $m$.

$$
x 2=x 1-\left(x^{m}-\bar{x}^{m}(k)\right)
$$

Thus the subblocks can be generated by using the equation 10 and the sequence should be chosen with the minimum value of PAPR for transmission [8]. Figure 3 depicts the OFDM structure with the modified PTS scheme.

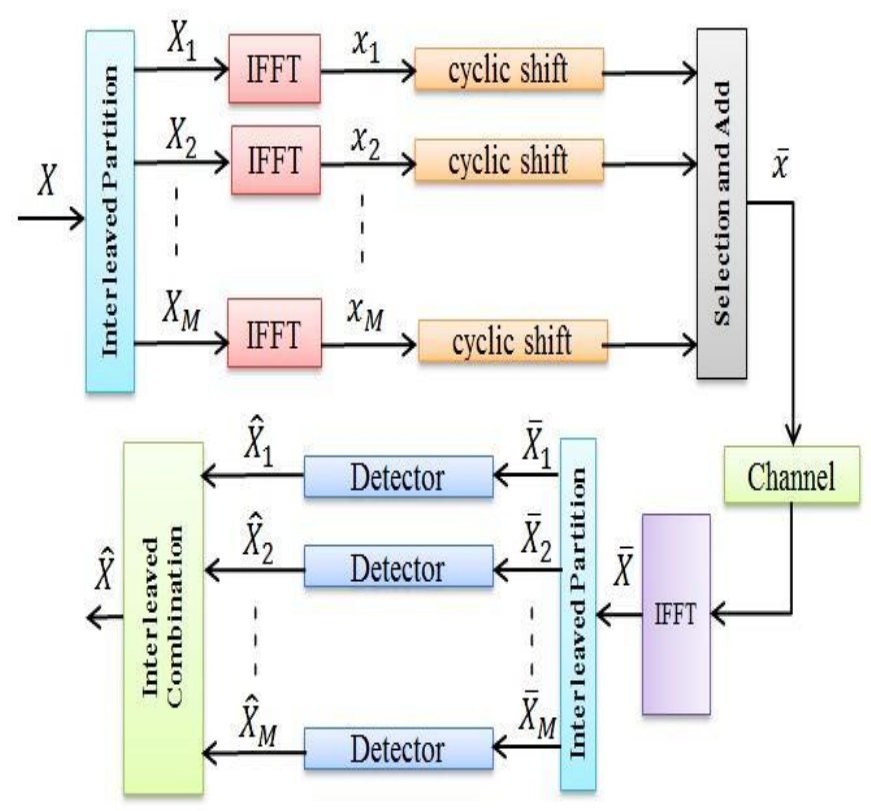

Figure 3. Block diagram of M-PTS Scheme

In this the property of cyclically shifting of IFFT is utilized which operates on each subblocks individually for shifting the number $\mathrm{k}$ as the cyclically shifting signal which have the eminent phase constellation in frequency domain and at the receiver, detector determines the shift number $\mathrm{k}$ according to the phase constellations for the signal received after FFT without using the side information. Thus the computational complexity is achieved by using Cooley-Tukey algorithm with this interleaved partition method. 


\subsection{Analysis of Computational Complexity for the C-PTS and the M- PTS}

The computational complexity for the C-PTS and the modified PTS has been analyzed for transmitter and the receiver.

\subsubsection{Computational Complexity for the Transmitter}

In this section the computational complexity of the proposed scheme is compared with the C-PTS and other low complexity PTS schemes.

When the number of subcarriers is $N=2^{n}$ and the oversampling factor is $L=2^{l}$. The numbers of complex multiplications and complex additions required for the conventional PTS scheme can be express as follows:

The $L N$-point IFFT requires $L N / 2 \log _{2}(L N)$ numbers of complex multiplication $\left(n_{m u l}\right)$ and $L N \log _{2}(L N)$ numbers of complex addition $\left(n_{\text {add }}\right)$ thus for the C-PTS the total $n_{m u l}$ and $n_{a d d}$ are given as $L N / 2 \log _{2}(L N / M)$ and

$L N \log _{2}(L N / M)+W^{(M-1)}(M-1) L N$

respectively.

While for the modified PTS scheme, the total $n_{m u l}$ and $n_{\text {add }}$ are given as $L N / 2 \log _{2}(L N / M)$ and $L N \log _{2}(L N / M)+W^{(M-1)} 2 L N$, respectively.

\subsubsection{Computational Complexity for the Receiver}

The computational complexity for both receivers of modified PTS and C-PTS are divided in two parts-

(i) A $L N$-point FFT operation and

(ii) The detector

The overall computational complexity for the C-PTS scheme is:

$n_{m u l}=L N / 2 \log _{2}\left(L^{2} N^{2} / M\right)+(Q+2) N$

$n_{\text {add }}=L N \log _{2}\left(L^{2} N^{2} / M\right)+W^{(M-1)}(M-1) L N+2 Q N$

While the overall computational complexity for the modified PTS scheme is:

$n_{\text {mul }}=L N / 2 \log _{2}\left(L^{2} N^{2} / M\right)+(Q+2) W N$

$n_{\text {add }}=L N \log _{2}\left(L^{2} N^{2} / M\right)+W^{(M-1)} 2 L N+2 Q W N$

The computational complexity reduction ratio (CCRR) of the modified PTS scheme over the C-PTS scheme is defined as $[8,9,10]$ :

$C C R R=\left(1-\frac{\text { Complexity of the modified PTS }}{\text { Complexity of the C }- \text { PTS }}\right) \times 100 \%$

In table 1 the comparison for the different values of CCRRs is shown in between C-PTS and the M-PTS for different values of $\mathrm{M} \& \mathrm{~N}$.
Table 1. Computational Complexity Reduction Ratio

\begin{tabular}{|l|l|l|}
\hline \multicolumn{1}{|c|}{ Comparison } & \multicolumn{2}{c|}{ CCRR } \\
\hline Subblock & $\mathrm{M}=8$ & $\mathrm{M}=16$ \\
\hline Complex Addition & $71.01 \%$ & $86.3 \%$ \\
\hline Complex Multiplication & $72.66 \%$ & $87.12 \%$ \\
\hline
\end{tabular}

\section{SIMULATION RESULTS}

The analysis has been done with the help of MATLAB and several simulations results are assess which describes that modified PTS shows better performance in comparison to C-PTS. In this QPSK modulation scheme is used. The evaluation of the performance is analysed in terms of complementary cumulative distribution function.

Table 2. Simulation Parameters

\begin{tabular}{|l|l|}
\hline PARAMETERS & VALUE \\
\hline Modulation & QPSK \\
\hline Number of subcarriers (N) & 256 \\
\hline Number of sub-blocks (M) & 8,16 \\
\hline Channel & AWGN \\
\hline Oversampling factor (L) & 4 \\
\hline $\begin{array}{l}\text { Phase factor possible values } \\
\text { (W) }\end{array}$ & $4(1,-1, \mathrm{j},-\mathrm{j})$ \\
\hline HPA model & SSPA \\
\hline
\end{tabular}

Figure 4 shows the Simulation Results of Conventional PTS.

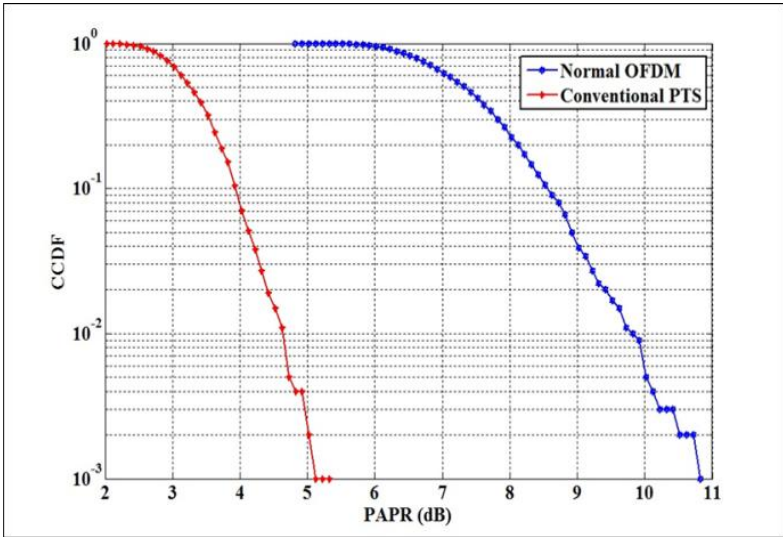

Figure 4. PAPR performance of C-PTS and normal OFDM

In Figure 5 comparison between the proposed modified PTS scheme (C-PTS) with conventional PTS scheme is shown and we found that CCDF of proposed C-PTS scheme is superior. 


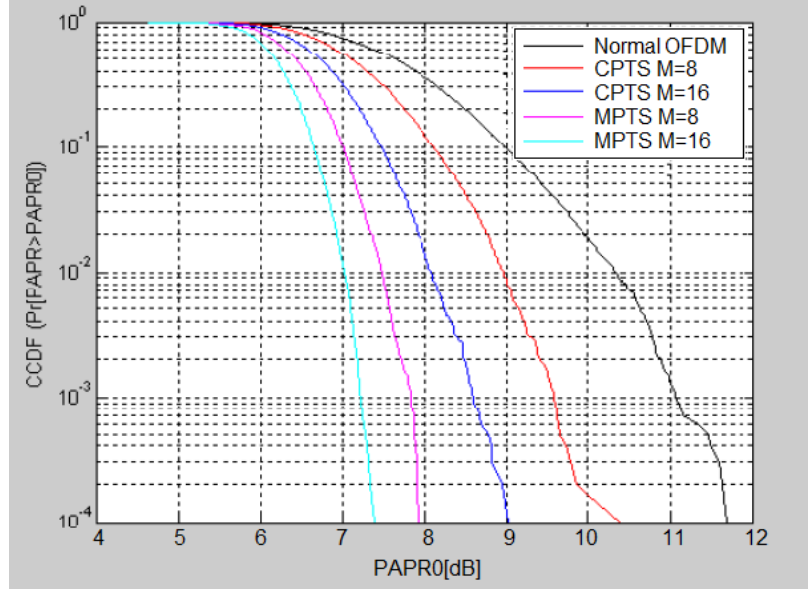

Figure 5. PAPR performance of MPTS, CPTS and normal OFDM for $N=256, M=8 \& 16$

\section{CONCLUSION}

In this paper, a modulated PTS scheme with reduced complexity has been proposed. In this approach cyclically shifting property of IFFT is used which doesn't require side information for the transmission. With this technique the complexity has been reduced in comparison with the conventional partial transmit sequence and the PAPR reduction performance is improved with increase in the number of sub-blocks $M$ due to increase in the number of values.

\section{REFERENCES}

[1] Jae Hong Lee Seung Hee Han, "An Overview of peakto-average power ratio reduction techniques for multicarrier transmission," IEEE Wireless Communications, pp. 56-65, April 2005.

[2] Yiyan Wu Tao Jiang, “An Overview: Peak-to-Average Power Ratio Reduction Techniques for OFDM Signals," IEEE transactions on broadcasting, vol. 54, no. 2, june 2008.

[3] Muller, S. H. and J. B. Huber, "OFDM with reduced peak-to average power ratio by optimum combination of partial transmit sequences," IEEE Electron. Lett., Vol. 33,368-369, Feb. 1997.

[4] S. H. Muller and J. B. Huber, "OFDM with reduced peak-to-average power ratio by optimum combination of partial transmit sequences," Electron. Lett., vol. 33, pp. 368-369, 1997.

[5] S. H. Han and J. H. Lee, "PAPR reduction of OFDM signals using a reduced complexity PTS technique," IEEE Sig. Proc. Lett., vol. 11, no. 11, pp. 887-890, Nov. 2004.

[6] L. J. Cimini Jr. and N. R. Sollenberger, "Peak-toaverage power ratio reduction of an OFDM signal using partial transmit sequences,"IEEE Commun. Lett., vol. 4, no. 3, pp. 86-88, Mar. 2000.

[7] Abdelsalam Sayed Ahmed,Mona Shokair,El Sayed El Rabaie, "PAPR Reduction for LFDMA using a reduced Complexity PTS Scheme," The online Journal on Electronics and Electrival Engineeing,Vol.04 No.02,pp. 524-530.

[8] L. Wang, O. Yuan and H. C. Chen, “A low-complexity peak-to average power ratio reduction technique for OFDM-based systems," IEEE VTC 2004-Fall, 2004, vol. 6, pp. 4380-4384.

[9] Ghassemi, A.; Gulliver, T.A., “A Low-Complexity PTS-Based Radix FFT Method for PAPR Reduction in OFDM Systems," IEEE Transactions on Signal Processing, vol.56, no.3, pp.11611166,March 2008.

[10] Pooria Varahram, Borhanuddin Mohd Ali, Senior Member, IEEE, "Partial Transmit Sequence Scheme with New Phase Sequence for PAPR Reduction in OFDM Systems", IEEE Transactions on Consumer Electronics, Vol. 57, No. 2, May 2011.

[11] Chenyang Yang,Gang Wu, Shaoqian Li, and Geoffrey Ye Li Taewon Hwang, "OFDM and Its Wireless Applications: A Survey," IEEE Transactions on Vehicular Technology, Vol. 58, No. 4, pp. 1673-1694, May 2009.

[12] Tao Jiang and Yiyan Wu, "An Overview: peak to average power ratio reduction technique for OFDM system," IEEE Transactions on Broadcasting, vol. 54, no.2,pp. 257-268, June 2008.

[13] Y.Wu and W. Y. Zou, "Orthogonal frequency division multiplexing: A multi-carrier modulation scheme," IEEE Trans. Consumer Electronics, Vol. 42, No. 3, pp. 392-399, August 1995.

[14] W. Y. Zou and Y. Wu, "COFDM: An overview," IEEE Trans. Broadcasting," Vol. 41, No.1, pp. 1-8, March 1995.

[15] Ramjee Prasad, "OFDM for Wireless Communications systems”, Artech House Publishers, 2004.

[16] Breiling, M., Muller-Weinfurtner, S. H., and Huber, J. B., "SLM peak- power reduction without explicit side information," IEEE Communications Letters, vol. 5, pp. 239-241, June 2001.

[17] R. W. Bauml, R. F. H. Fischer, and J. B. Huber, "Reducing the peak-to- average power ratio of multicarrier modulation by selected mapping," Electron. Lett., vol. 32, pp. 2056-2057, Oct. 1996.

[18] G.Tong Zhou and Liang Peng, "Optimality condition for Selected Mapping in OFDM ," IEEE Transactions on signal processing, vol. 54,no.8, pp. 3159-3165, Aug. 2006.

[19] Seung Hee Han and Jae Hong Lee, “ An overview of peak-to-average power ratio reduction techniques for multicarrier transmission," IEEE Wireless Communications, Vol.12,No.2, pp.56 - 65, 2005.

[20] D Wulich, and L Goldfeld, "Reduction of peak factor in orthogonal multicarrier modulation by amplitude limiting and coding," IEEE Transactions. Communication, vol. 47, no. 1, pp 18-21, January 1999. 